\title{
Gravity Balancing Flexure Spring Mechanisms for Shoulder Support in Assistive Orthoses
}

This paper was downloaded from TechRxiv (https://www.techrxiv.org).

\section{LICENSE}

CC BY 4.0

SUBMISSION DATE / POSTED DATE

$21-03-2021 / 31-03-2021$

\section{CITATION}

Tschiersky, Martin; Hekman, Edsko E. G.; Herder, Just L.; Brouwer, Dannis M. (2021): Gravity Balancing Flexure Spring Mechanisms for Shoulder Support in Assistive Orthoses. TechRxiv. Preprint. https://doi.org/10.36227/techrxiv.14256044

$\mathrm{DOI}$

10.36227/techrxiv. 14256044 


\title{
Gravity Balancing Flexure Spring Mechanisms for Shoulder Support in Assistive Orthoses
}

\author{
Martin Tschiersky, Edsko E. G. Hekman, Member, IEEE, Just L. Herder Member, IEEE, and Dannis M. Brouwer
}

\begin{abstract}
Passive shoulder supports show large potential for a wide range of applications, such as assisting activities of daily living and supporting work-related tasks. The rigid architectures of currently available devices, however, may pose an obstacle to finding designs that offer low protrusion and close-to-the-body alignment. This study explores the use of mechanisms that employ a flexible element which connects the supported arm to an attachment at the back and acts as energy storage, transmission and part of the load bearing structure. Based on the synthesis method explained in this paper, we conducted a large scope investigation into possible flexure-based mechanism topologies. Many potential designs were discovered and are presented, categorized and compared. Two promising designs were developed into prototypes that were built and tested on a dedicated test bench. These mechanisms reduce the necessary moment to lift the arm by more than $80 \%$ throughout $85 \%$ of the range of motion, while staying within $18 \mathrm{~cm}$ and $10 \mathrm{~cm}$ distance from the body, respectively. Our study indicates that, due to its lower protrusion and interface loads, a design with a tapered flexure connecting the upper arm via a hinge to a spring loaded slider at the back offers the most promising solution.
\end{abstract}

Index Terms-gravity balancing, wearable device, assistive orthosis, shoulder support, flexure spring.

\section{INTRODUCTION}

W EARABLE upper-limb assistive devices are used to facilitate functional arm movements. By providing supporting forces and moments to the wearer they reduce the amount of muscle exertion necessary to perform manual tasks. While potentially beneficial for a wide range of use cases, they are currently mostly used and investigated in two main areas. The first pertains to people suffering from deficient motor function who can use such devices to restore their ability to perform activities of daily living (ADLs), and thus reduce their reliance on external help [1], [2]; The second concerns workers who are exposed to physically demanding tasks, and who may use them to mitigate their risk of work-related musculoskeletal disorders (MSDs) [3].

Assistive devices for the upper-limb can be broadly characterized as either end-effectors or orthoses. The latter can be

This project has received funding from the European Union's Horizon 2020 research and innovation programme under grant agreement No. 688857 (SoftPro). The content of this publication is the sole responsibility of the authors. The European Commission or its services cannot be held responsible for any use that may be made of the information it contains.

M. Tschiersky and D.M. Brouwer are with the Chair of Precision Engineering, University of Twente, Enschede, 7500 AE, The Netherlands (e-mail: m.tschiersky@utwente.nl).

E.E.G. Hekman is with the Department of Biomechanical Engineering, University of Twente, Enschede, 7500 AE, The Netherlands.

J.L. Herder is with the Department of Precision and Microsystems Engineering, Delft University of Technology, Delft, 2628 CD, The Netherlands. subdivided into rigid exoskeletons and soft exosuits. Within each category further distinctions can be made based upon the applied actuation scheme [1], [2], [4], [5], [6], [7]. Active exoskeletons and, more recently, exosuits are being extensively investigated in academic research. However, commercially available devices for assisting ADLs are predominantly passive end-effectors [1], while work assist devices that support the upper limb are exclusively passive exoskeletons [3].

Considering passive orthoses that provide dynamic shoulder support, five potentially fully wearable devices designated for assisting ADLs exist to our knowledge. The A-Gear [8], Panto-Arm Exo [9] and Springwear [10] are academic research prototypes, while the EksoUE [11] and Wilmington Robotic Exoskeleton (WREX) [12], [13] are commercially available devices. On the other hand, in recent years several companies have entered the market with wearable work assist exoskeletons. Examples are: the Airframe [14], [15], EksoVest and EVO [11], [16], MATE [17], [18], PAEXO [19], [20], ShoulderX [21], [22] and SkelEx [23], [24].

All the above mentioned are passive devices that work by applying forces and moments to the wearer's upper-limb and thereby counter the gravity loads acting upon it. Thus, the amount of force the user has to exert in order to lift the arms is reduced. To this end, these exoskeletons employ rigid link mechanisms that contain energy storing elements like tension and compression springs [14], [17], [21], gas springs [9], [11], rubber bands [8], [10], [12], [19] and leaf spring flexures [23] in combination with transmission elements like rigid linkages [8], [9], [10], [12], [19], [23], cable-pulley configurations [14], [21], gears [17] and cams [11] which together create the desired mechanical behavior. These types of mechanisms, however, imply an exoskeleton design in which a rigid frame and rigid links are used to interface with the wearer's body. Such being the case, additional links, joints and sliders are added to make the rigid structure adjustable and comply with the shoulder kinematics, increasing the complexity and number of parts necessary. Furthermore, to avoid potentially harmful collision moving parts are required to maintain a safe distance to the body, often leading to significant protrusion.

With the research presented in this paper we attempt to address these issues. In our design approach we connect a flexible element resembling a curved flexure with variable thickness between the users back and upper arm. Similar to the SkelEx device, this flexure acts as both the energy storing element and as a part of the frame. However in contrast, the flexure is designed to be more compliant and exhibit large 
deformations. Furthermore, no additional links between the arm and the back are used to prescribe its deformation and to create the gravity balancing properties. Instead, by utilizing the natural shoulder kinematics, the gravity balancing behavior emerges from the shape of the flexure in combination with the kinematic constraints prescribed at each end. Thus, the flexure integrates functions of energy storage, transmission and load bearing structure.

Using this approach we sought to obtain devices that are structurally more flexible than current exoskeletons, while preserving comparable external load bearing capabilities. Using the gain in flexibility we aimed to bring the device closer to the wearer's body, as due to the yielding nature of the flexure we hypothesized that the wearer would not incur any harm in the event of a collision. Furthermore, the flexure may offer enough compliance to allow motion in the non-actuated directions, reducing the need for dedicated mechanisms to comply with the degrees of freedom of the shoulder. Together with the above described function integration this may reduce the overall complexity and lead to a smaller part count.

To find appropriate designs, we created a computational model which encompasses the flexible element and the wearer to simulate the mechanical behavior of the entire system and to determine the distance between the two. Using this model we optimized flexure shapes for various support conditions at each end, to find flexure spring mechanisms that offer both adequate gravity balancing support and close-to-the-body alignment.

The approach is based on our previous work on gravity balancing flexure springs for an assistive elbow orthosis [25] and similar work on gravity balancing flexure mechanisms within [26], [27] and without [28], [29], [30] the context of assistive devices. However, we expanded the method by introducing a new use case, multiple design objectives and more complex spatial constraints, in which collision with the body is to be avoided while remaining in close proximity to it. Furthermore, we conducted an extensive investigation into the effects of using different kinematic support conditions at each end of the flexure, representing sliders, hinges and combinations thereof with zero, finite or infinite stiffness.

Following this introduction, in the Synthesis section, we describe the applied methods by which we obtained gravity balancing flexure spring mechanisms for the several different kinematic support conditions. From these we selected two support conditions for further investigation, from which we derived two prototype designs. The subsequent Experiment section deals with the physical validation of the prototypes on a dedicated test bench. The outcomes of both the synthesis and experiment are presented in the Results section and reflected upon in the Discussion section. Lastly we provide our key insights in the Conclusion section.

\section{SYNTHESIS}

\section{A. Model Topology}

For the design of the flexure mechanisms, we consider the flexible element with its end supports, as well as the torso and right arm of the wearer. The schematic model topology is shown in Figure 1. Mechanically, the shoulder joint is reduced to a pair of revolute joints which are connected to

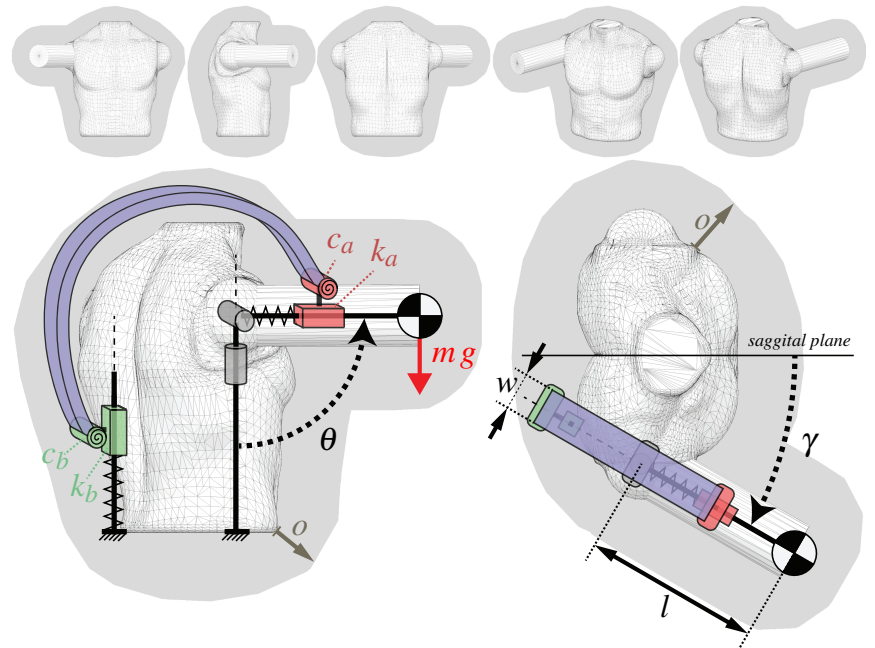

Fig. 1. Model topology. The triangulated meshes for the torso and right upper arm are shown in white, the offset mesh volume is indicated in gray. The top figures show the meshes from several perspectives. The bottom left figure is a view perpendicular to the arm plane. The bottom right figure is a view from the top. Rigid links are shown as black bold lines. Their axes are indicated by dashed lines. The prismatic and revolute joints are drawn in gray for the shoulder, red for the arm interface and green for the back interface. The flexible element, i.e. the flexure, is shown in blue.

each other and coincide with the position of the glenohumeral joint. The joints are oriented perpendicular to each other and their motion corresponds to the upper arm elevation $\theta$ and horizontal flexion/extension $\gamma$, respectively. Their position in space is fixed with respect to ground. Endo/exorotation of the arm is not considered. The arm itself is treated as a rigid link with a mass $m$ located at the center of gravity and is connected to the pair of revolute joints at its proximal end. The position of the mass is fixed as elbow and hand motion are not considered.

The flexible element is treated as a prismatic beam with rectangular cross-section and varying thickness along its length. It is connected to a point at the back of the wearer at one end, travels above the top of the right shoulder, and connects to the upper arm section of the arm link at the other end. At each interface point the flexure connects via a slider/hinge combination. The slider at the back allows motion along the vertical axis and the slider on the arm motion along the arm axis. The orientation of the hinges is perpendicular to the arm elevation plane. By setting the stiffnesses of these kinematic elements, we can model each connection as either a clamp, slider, hinge or a combination of both slider and hinge. In addition, when using finite stiffness values, each element can also act as a spring with a translational stiffness $k$ or rotational stiffness $c$, respectively.

The volumes of the torso and right upper arm are represented by triangulated meshes. The position and orientation of the torso mesh is fixed, while the upper arm mesh orientation follows the arm link. For each mesh a corresponding offset mesh with a uniform offset $o$ prescribes the space in which the flexible element is allowed to operate.

\section{B. Design objectives}

The primary goal of this study is to find the ideal shape and thickness distribution of the flexible element and the ideal 
joint stiffness values for each prescribed kinematic support condition, such that the resulting mechanism balances the mass $m$ on the arm link, while not colliding with but staying within close proximity to the wearer. This should be achieved while featuring a small flexure width to reduce the overall size, protrusion and out-of-plane stiffness. Furthermore, to allow a design in which the flexible element can be directly connected to the arm, parasitic forces and moment that do not contribute to the gravity balancing behavior should be minimal. To this end, four metrics are used to assess the quality of a design.

Gravity balancing quality, which is the primary objective, is defined as the ability of the flexure mechanism to counteract the effect of gravity on a mass $m$, such that the moment $M$ at the shoulder elevation joint is zero for any elevation angle $\theta$ in the range from 0 to $\pi$. For our model, the moment caused by gravity can be expressed as:

$$
M_{g}=-a_{g} \sin (\theta) \quad a_{g}=m g l
$$

where $a$ is the moment amplitude, $g$ the gravitational acceleration and $l$ the distance between the center of gravity and the shoulder joint. Consequently, the moment $M_{f}$ exerted by the flexure mechanism has to be the inverse. However, we divide $M_{g}$ and $M_{f}$ by their respective maximum amplitude and obtain the relation for the ideal case:

$$
\frac{M_{f}}{a_{f}}-\sin (\theta)=0 \quad a_{f}=\max \left(\left|M_{f}\right|\right)
$$

The gravity balancing quality can now be expressed by the cumulative error in this equation for the considered range of motion. Since we are discretizing the elevation angle in both our simulations and experiments we chose to use the normalized mean absolute error (NMAE):

$$
\boldsymbol{\delta}=\sum_{i=0}^{n} \frac{\left|\frac{M_{f, i}}{a_{f, i}}-\sin \left(\theta_{i}\right)\right|}{n}
$$

where $i$ is a data point and $n$ the total number of data points.

The second metric is the ratio between the maximum moment amplitude and the flexure width, serving as an indicator for the relative strength of the design. We define this as:

$$
\boldsymbol{\epsilon}=\frac{a_{f}}{w}
$$

The third metric is the maximum protrusion of the flexible element from the body. It is measured as the maximum distance between the left or right edge of the flexure's neutral plane, i.e. mid-plane, to the respective closest point on the combined torso and arm mesh.

Finally, the fourth metric is the ratio between the maximum amount of shear force at the arm interface and the maximum moment amplitude. The shear force is defined as the force component at the arm interface parallel to the arm axis. It is defined positive when pointing in distal direction, and negative when pointing in proximal direction.

\section{Application requirements}

We intend the device to assist in the lifting of the arms when no additional payload is considered. This type of support is rather task independent and would potentially benefit both prior mentioned use case scenarios of assisting ADLs and physically demanding work-related tasks. The device shall work for the entire range of motion of the shoulder from the relaxed arm position at $\theta=0$ to the raised position at $\theta=\pi$.

In order to provide adequate gravity compensation, both the balancing quality and the balancing quantity have to be sufficient. Based on the experience from our previous work [25] we determined that a NMAE $\leq 0.05$ would constitute a good balancing behavior. Regarding the quantity, we calculated that based on the mean body segment data of female and male subjects [31] a moment amplitude of $9.16 \mathrm{Nm}$ would be necessary to fully compensate for the gravity loads acting upon the arm. Based on this number we chose a target moment amplitude of $10 \mathrm{Nm}$, offering a margin for customization.

The protrusion of the device from the body should be minimal. A study on passive assistive orthoses found that devices should stay within $30 \mathrm{~mm}$ from the body, and to be wearable underneath clothing even below $20 \mathrm{~mm}$ [2]. However, considering existing passive assistive shoulder supports, these figures seem overly ambitious. Therefore, we did not derive a hard requirement but rather treat this design aspect as a main point of investigation.

It is known from literature that contact pressure and shear applied to the skin should be minimal in order to comfortably use a device for extended periods of time [32]. However, we found no practical threshold values in literature that pertain to our use case. Therefore, we also left this aspect open to investigation.

\section{Additional specifications}

This study is limited to planar mechanism designs. This was done in order to reduce the number of design parameters for the optimization. In addition, to reduce simulation effort the arm elevation is only investigated in one plane. To this end, an elevation plane with a horizontal extension angle of $\gamma=\frac{\pi}{6}$ towards the saggittal plane was chosen.

Regarding the protrusion criterion, heightened attention was placed on arm elevations between $\theta=0$ and $\theta=\frac{\pi}{2}$, since a low protrusion was deemed more critical for ADL's of which most only require this range of motion [33].

A model of a $183 \mathrm{~cm}$ tall male subject was used for reference to determine collision with and protrusion from the torso. The upper arm was assumed to be a cylinder with $10 \mathrm{~cm}$ diameter and $30 \mathrm{~cm}$ length.

\section{E. Computational model}

For analysis we used the flexible multibody dynamics software package SPACAR [34]. The entire mechanism was modeled using hinges and finite two-node beam elements which feature geometric nonlinear behavior and flexibility formulated in six discrete deformation modes. At the shoulder a hinge corresponding to the arm elevation is fixed to the ground at one end and connected to the arm link at the other. Its position marks the origin of the Cartesian coordinate system. The arm link is modeled as a beam with one released deformation mode that corresponds to its elongation. As it can 
only permit motion along its axis and otherwise acts rigid, it can be used to model a slider with a linear stiffness $k_{a}$. A small perpendicular rigid beam extends from the arm link, i.e. the arm axis, to a position $2 \mathrm{~cm}$ above the surface of the arm cylinder. At this position, which serves as the interface point to the flexure, a hinge with a rotational stiffness $c_{a}$ is placed to connect arm and flexure. At the back a similar construction is used to model the vertical slider with linear stiffness $k_{b}$ and the back hinge with rotational stiffness $c_{b}$, though in this case no extension beam is used. Setting the stiffnesses to infinite, zero or finite values enables the modeling of clamps, hinges, sliders and slider/hinge combinations at each interface point, with and without springs acting in parallel to the joints. A point mass of $0.2 \mathrm{~kg}$ was a assigned to the back interface to account for the weight of the mechanical components.

The flexure which connects the hinge at the back to that on the arm is modeled as a chain of multiple beam elements that allow initial pre-curvature [35] and are fully released, thus exhibit full flexible behavior. By definition the chain starts at the back and ends at a point above the shoulder joint. The initial position of the back slider and hinge are set according to the start position of the beam. Similarly, the initial length of the arm link and the initial elevation angle of the shoulder hinge are set such that the arm interface point coincides with the end of the flexure. As a consequence and since no pre-tension is applied, the elevation angle that corresponds to the unstressed position of mechanism depends on the flexure design.

The mesh of the torso is derived from a CAD model of a full human body. The head, arms and lower extremities have been removed and spherical volumes were placed at the position of the glenohumeral joints. The position of the right glenohumeral joint is made to coincide with the shoulder hinge. The torso is rotated about the origin to set the relative angle $\gamma=\frac{\pi}{6}$ between the plane of the mechanism and the torso's saggital plane. The cylinder mesh representing the upper arm is fixed to the origin at its proximal end and is aligned with the upper arm link. The offset meshes for the torso and arm were generated from the original collision detection meshes using a voxel-based approach, and placed and oriented in the same way.

\section{F. Simulation}

To analyze a mechanism design, a kinetostatic analysis is performed. The model is first moved from its arbitrary initial position to the fully raised position at $\theta=\pi$. Then the shoulder hinge controlling arm elevation is rotated from $\theta=\pi$ to $\theta=0$ in a number of predefined load steps $n$. For each load step the moment at the shoulder, as well as the forces and moments at the interface points are evaluated. Furthermore, based on the node positions and orientations as well as the width of each flexible beam element constituting the flexure, contact nodes are generated that lie on the vertices of each beam's neutral plane. These contact nodes are used to determine whether a beam lies within the torso mesh, arm mesh or their respective offset meshes. Based on that information, the protrusion distance from or the penetration depth into the torso and arm mesh is measured for each node.

\section{G. Parameterization}

In order to optimize the mechanism its components have to be parameterized. The neutral axis of the flexure, and thus the node positions and orientations for each beam element, is obtained by constructing a Frenet ribbon with minimum torsion from a natural cubic spline curve which interpolates a number of control points. The position of the first control point is given explicitly by its coordinates, while all other points are obtained through a linkage chain formulation similar to the one used in [25]. To this end, the first two parameters of the parameter set are the horizontal position $s_{x}$ and the vertical position $s_{z}$ of the starting point at the back. These are followed by a number of link lengths $l$ and angles $\varphi$ corresponding to the number of links in the linkage chain and consequently to the number of additional control points. An illustration of such a linkage chain can be found in [25], though for this study instead of relative angles between links absolute angles towards the ground were used. The thickness of each beam element is obtained by linear interpolation of the thickness parameters $t$ over the beam nodes. The mean value of the thickness values at the two nodes of each beam element yields its thickness in the model. An illustration of this can also be found in [25], though instead of multipliers absolute thickness values were used. Lastly, depending on the investigated support conditions for the flexure, the stiffness values of the slider and hinge elements are appended, yielding a parameter set in the form:

$$
\begin{aligned}
\mathbf{x}=[\quad & {\left[s_{x}, s_{z}\right],\left[l_{1}, \ldots, l_{n}\right],\left[\varphi_{1}, \ldots, \varphi_{n}\right], } \\
& {\left.\left[t_{1}, \ldots, t_{n}\right],\left[k_{a}, c_{a}, k_{b}, c_{b}\right]\right] }
\end{aligned}
$$

\section{H. Optimization}

To find optimal parameter sets a two-step optimization procedure was conducted. First the mechanisms are optimized towards a high balancing quality, i.e. to minimize the error $\delta$ as defined in Equation 3. In the second step, this error is limited to $\delta \leq 0.05$, while the relative strength of the mechanism is optimized towards a maximum value of $\epsilon$ as defined in Equation 4. With the exception of the redefinition of $\delta$ from objective to constraint, both optimizations are subject to the same set of bounds and constraints. The constraints are implemented as inequality constraints, denoted $\mathbf{c}$, and soft constraints, i.e. penalties $\mathbf{p}$. The inequality constraints $\mathbf{c}$ are evaluated for the initial mechanism state, before running the SPACAR simulation. If any of these produce a value $>0$, the simulation is skipped and an error value returned. The penalties $\mathbf{p}$, similar to the objectives $\delta$ and $\epsilon$, are evaluated after the simulation. Their values are multiplied with a penalty factor $k_{p}=1 \mathrm{e} 5$ and added to the cost function. The two cost functions can be written as:

$$
\begin{gathered}
f_{1}(\mathbf{x})=\boldsymbol{\delta}+k_{p} \mathbf{p}_{(1-6)} \\
f_{2}(\mathbf{x})=1 / \epsilon+k_{p} \mathbf{p}_{(1-7)} \\
\text { subject to } \mathbf{c}_{(1-7)} \leq 0
\end{gathered}
$$

The first two constraints $\mathbf{c}_{(1)}$ and $\mathbf{c}_{(2)}$ check, whether the distal end of the arm is pointing upwards with a maximum 
deviation of $\alpha=\frac{\pi}{9}$ from vertical.

$$
\begin{aligned}
& \mathbf{c}_{(1)}=\cos \left(\theta_{\text {init }}\right) \\
& \mathbf{c}_{(2)}=\left|\sin \left(\theta_{\text {init }}\right)\right|-\sin (\alpha)
\end{aligned}
$$

where $\theta_{\text {init }}$ is the initial angle of the arm link.

The next two constraints $\mathbf{c}_{(3)}$ and $\mathbf{c}_{(4)}$ check, whether the flexure attaches to the upper arm within a specified distance range $1=\left[\begin{array}{lll}0.05 & \ldots & 0.3\end{array}\right] \mathrm{m}$ from the shoulder.

$$
\begin{aligned}
& \mathbf{c}_{(3)}=t_{\min }-t \\
& \mathbf{c}_{(4)}=t-t_{\max }
\end{aligned}
$$

where $t$ is the length of the arm link, i.e. of the respective beam element in the SPACAR model.

For the constraint $\mathbf{c}_{(5)}$ a self-intersection test is performed on the flexure. To this end, each line segment given by the end nodes of the beam elements constituting the flexure is checked for intersection with all other line segments. This procedure, which is described in detail in [25], yields the total number of self-intersections $\mathrm{n}_{\times}$.

$$
\mathbf{c}_{(5)}=\mathrm{n}_{\times}
$$

Constraint $\mathbf{c}_{(6)}$ determines whether the beam penetrates either the torso or the arm mesh. Each contact node $j$ is assigned a value of $\mathrm{n}_{\in}=1$, if it lies within at least one of the two meshes, and $n_{\in}=0$ if not. The sum of $n_{\in}$ for all contact nodes yields the the constraint value.

$$
\mathbf{c}_{(6)}=\sum_{j=0}^{m} \mathrm{n}_{\epsilon}
$$

Analogously, the constraint $\mathbf{c}_{(7)}$ determines whether the beam lies outside both the torso and arm offset meshes. Each contact node is assigned a value of $\mathrm{n}_{\notin}=1$ if it lies outside both the torso and arm offset mesh, and $\mathrm{n}_{\notin}=0$ if not. However, in the scope of this constraint only the first four contact nodes of the beam element at the back interface are checked, to ensure the flexure starts within the protrusion limit.

$$
\mathbf{c}_{(7)}=\sum_{j=0}^{4} \mathrm{n}_{\notin}
$$

The first three penalties directly correspond to the constraints $\mathbf{c}_{(3)}, \mathbf{c}_{(4)}$ and $\mathbf{c}_{(5)}$, whereby the respective constraint values are evaluated for each load step $i$ and added in case they are positive. Penalties $\mathbf{p}_{(1)}$ and $\mathbf{p}_{(2)}$ shall prevent the interface point from sliding outside the specified range from the shoulder, when the arm link acts as a slider.

$$
\begin{array}{lll}
\mathbf{p}_{(1)}=\sum_{i=0}^{n} t_{\text {min }}-t & \forall & t_{\text {min }}-t>0 \\
\mathbf{p}_{(2)}=\sum_{i=0}^{n} t-t_{\text {max }} & \forall & t-t_{\text {max }}>0
\end{array}
$$

Penalty $\mathbf{p}_{(3)}$ shall prevent self-intersections throughout the full deformation range of the flexure.

$$
\mathbf{p}_{(3)}=\sum_{i=0}^{n} \mathrm{n}_{\times}
$$

Penalties $\mathbf{p}_{(4)}$ and $\mathbf{p}_{(5)}$ are an extension to the constraints $\mathbf{c}_{(6)}$ and $\mathbf{c}_{(7)}$. In addition to evaluating whether a node lies within the torso and arm mesh or outside the offset meshes, the penetration depth and protrusion distance are evaluated for each respective node, as to provide a more continuous change in the cost function. For both $\mathbf{p}_{(4)}$ and $\mathbf{p}_{(5)}$ this evaluation is carried out for all contact nodes. However, while the penetration depth is considered for the entire range of motion, the protrusion is only taken into account for the lower elevation angles $\theta \leq \frac{\pi}{2}$ which are most critical for ADLs [33].

$$
\begin{array}{llll}
\mathbf{p}_{(4)}=\sum_{i=0}^{n} \sum_{j=0}^{m}|d| & & \mathrm{n}_{\in}=1 \\
\mathbf{p}_{(5)}=\sum_{i=n / 2}^{n} \sum_{j=0}^{m} d & & \mathrm{n}_{\notin}=1
\end{array}
$$

where $d$ is the shortest distance to either the torso or the arm mesh and index $j$ denotes each contact node. By convention, $d$ is signed negative when pointing inside the mesh and positive when pointing outside.

The last regular penalty $\mathbf{p}_{(6)}$ returns the value by which the von Mises stress exceeds the stress limit $\sigma_{\text {lim }}$. The von Mises stress in each beam element constituting the flexure is determined for each load step and the highest overall value assigned to $\sigma_{\text {Mises }}$.

$$
\begin{array}{r}
\mathbf{p}_{(6)}= \begin{cases}0, & \text { if } \sigma_{\text {Mises }}-\sigma_{\text {lim }} \leq 0 \\
\sigma_{\text {Mises }}-\sigma_{\text {lim }}, & \text { if } \sigma_{\text {Mises }}-\sigma_{\text {lim }}>0\end{cases} \\
\sigma_{\text {lim }}=\frac{\sigma_{t}}{\text { FOS }}
\end{array}
$$

where $\sigma_{t}$ is the tensile stress of the flexure material and $F O S=1.2$ the applied factor of safety.

As stated previously the objective $\delta$ of the first optimization is turned into a penalty for the second optimization. To this end, a conditional extra penalty $\mathbf{p}_{(7)}^{*}$ applies only during that second optimization.

$$
\mathbf{p}_{(7)}^{*}= \begin{cases}0, & \text { if } \quad \boldsymbol{\delta}-\delta_{\text {lim }} \leq 0 \\ \boldsymbol{\delta}-\delta_{\text {lim }}, & \text { if } \quad \boldsymbol{\delta}-\delta_{\text {lim }}>0\end{cases}
$$

where $\delta_{\text {lim }}=0.05$ is the limit on the gravity balancing error.

The two cost functions $f_{1}(\mathbf{x})$ and $f_{2}(\mathbf{x})$ are optimized sequentially using the particleswarm() solver from the MATLAB Global Optimization Toolbox, whereby the optimized parameter set of the first optimization serves as the initial point for the second optimization.

\section{Investigation scheme}

Using this optimization routine mechanism designs for every feasible combination of support conditions were optimized. First we investigated all combinations of support conditions, when considering only approximately zero or infinite stiffness at each joint. Hence, no joint stiffnesses parameters were optimized. However, since these designs rely on storing all their energy in the flexure, only designs with a mobility of the flexure of $\leq 0$ were considered. Consequently, combinations with $\geq 3$ degrees of freedom and with two sliders have been excluded. The latter, is due to the kinematic singularity that 
TABLE I

OVERVIEW OF FEASIBLE SUPPORT CONDITIONS

\begin{tabular}{|c|c|c|c|c|c|}
\hline & \multirow[b]{3}{*}{ DOF } & \multicolumn{4}{|c|}{ Back } \\
\hline & & Clamp & Hinge & Slider & $\begin{array}{l}\text { Slider/ } \\
\text { Hinge }\end{array}$ \\
\hline & & 0 & 1 & 1 & 2 \\
\hline Clamp & 0 & $\checkmark$ & $\checkmark$ & $\checkmark$ & $\checkmark$ \\
\hline Hinge & 1 & $\checkmark$ & $\checkmark$ & $\checkmark$ & $(\checkmark)^{\star}$ \\
\hline Slider & 1 & $\checkmark$ & $\checkmark$ & $(\checkmark)^{\star}$ & $(\checkmark)^{\star}$ \\
\hline Slider/Hinge & 2 & $\checkmark$ & $(\checkmark)^{\star}$ & $(\checkmark)^{\star}$ & $(\checkmark)^{\star}$ \\
\hline
\end{tabular}

occurs when both sliders are parallel. This left 10 feasible out of 16 possible combinations.

Next, we investigated all combinations of support conditions when a stiffness parameter is assigned to each released joint, i.e. when considering only a finite or infinite stiffness at each joint. Each assigned stiffness parameter becomes part of the parameter set and thus is optimized along the with the flexure shape. In this case no mobility issues arise making all combinations feasible. However, since the case with infinite stiffness in all joints is the same as before only 15 additional combinations are considered, making it in total 25 different support conditions that have been investigated. An overview of the investigated support conditions is given in Table I.

For each of the different support conditions the optimization routine was conducted at least 51 times and on average 65 times. During this process the maximum allowed protrusion, affecting the offset mesh and thus penalty $\mathbf{p}_{(5)}$, was alternated between the values $O=[75,100,150] \mathrm{mm}$. Based on this broad investigation the combinations of support conditions that yielded feasible results were identified.

These feasible mechanisms were then categorized regarding their kinematic behavior at each interface, and for each category two representative designs were chosen and dimensioned. PA2200 with a Young's modulus of $E=1.7 \mathrm{GPa}$, a tensile stress of $\sigma_{t}=50 \mathrm{MPa}$ and a density of $\rho=930 \mathrm{~kg} / \mathrm{m}^{3}$ was used as flexure material, and the maximum flexure thickness was limited to $25 \mathrm{~mm}$. By adjusting the flexure width and joint stiffnesses, a moment amplitude of $10 \mathrm{Nm}$ was set for every example mechanism. This provided a comparison between the different categories of feasible mechanisms. Two of those categories have been selected for further investigation. For these more targeted optimizations were conducted, yielding the improved flexure mechanism designs that underwent experimental evaluation.

\section{EXPERIMENT}

Following the investigation scheme physical prototypes for the two improved flexure mechanism designs, labeled $\mathbf{A}$ and $\mathbf{B}$, were made and tested on a designated test bench. The test bench was designed to emulated the conditions in the simulation, and thereby validate both the angle-dependent stiffness behavior and the alignment with the body.

Due to the different support conditions, prototype $\mathbf{A}$ and prototype $\mathbf{B}$ require different test bench configurations. The test bench as configured for prototype $\mathbf{B}$ is shown in Figure 2, while the configuration for prototype $\mathbf{A}$ is shown in the Results section Figure 6. For the structural components $40 \times 40 \mathrm{~mm}$

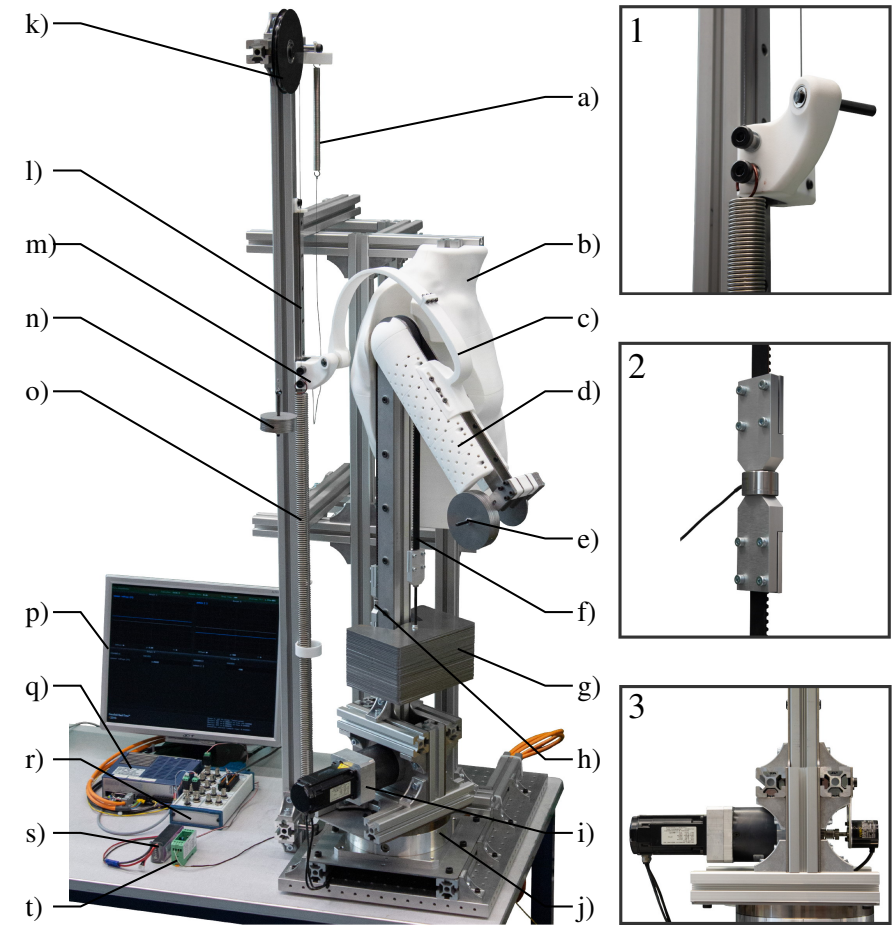

Fig. 2. Experimental setup, configuration B. a) secondary spring (connected to back of shuttle via string), b) torso shell, c) flexure specimen (prototype B), d) arm shell, e) balance weight, f) timing belt, g) counterweight, h) force sensor assembly, i) actuator (motor and gear), j) rotary stage, k) pulley, 1) linear guide, m) shuttle assembly, n) shuttle counterweight, o) primary spring, p) Simulink Real-Time scope, q) motor driver, r) NI adapter, s) battery, t) measuring amplifier. Detail 1: shuttle assembly without specimen; Detail 2: force sensor assembly; Detail 3: auxiliary view showing the position of the encoder, connected to the actuator output via a bellows coupling.

aluminum profiles and $3 \mathrm{~mm}$ steel plate were used, while a $25 \mathrm{~mm}$ thick aluminum plate serves as the base. The tested flexure prototypes, the arm and torso shells, as well as the hub of the shuttle assembly are made from laser sintered PA2200. Due to size limitations imposed by the laser sintering machine both flexures had to be split into two parts.

The arm consists of two parallel steel plates to which two shells with mounting holes are attached. At the distal end both plates are connected, and an adjustable balance weight is fixed to set the moment amplitude. The weight can be shifted to align the arm's center of gravity with the arm axis.

At the proximal end both plates are connected to a timing pulley which is mounted to the top of a pole via two ball bearings. Below the pole a second timing pulley is located and fixed to the output of a Neugart PLE080 gearbox which is driven by a Kollmorgen AKM22C motor. The motor is powered and controlled via a Kollmorgen S20260-VTS servo drive. Opposite to the gearbox a YUMO E6B2-CWZ3E digital quadrature encoder is connected to the same output through a bellows coupling.

Both timing pulleys are connect via an open timing belt. The belt is clamped to the lower pulley, runs over the top pulley and extends back down where it connects to an adjustable counterweight. In between the two pulleys the belt is interrupted by an assembly consisting of two clamps and a ME-Meßsysteme KM26z load cell. The signals from the load cell are amplified by a ME-Meßsysteme GSV-11H 010-5/20/2 measuring amplifier, using a battery for power supply. 
TABLE II

PERFORMANCE METRICS

\begin{tabular}{|c|c|c|c|c|c|}
\hline & & $\begin{array}{l}\text { Balancing } \\
\text { Error } \delta\end{array}$ & $\begin{array}{l}\text { Relative } \\
\text { Strength } \epsilon\end{array}$ & $\begin{array}{l}\text { Maximum } \\
\text { Protrusion }\end{array}$ & $\begin{array}{l}\text { Relative } \\
\text { Shear Force }\end{array}$ \\
\hline & & - & $\mathrm{Nm} / \mathrm{cm}$ & $\mathrm{cm}$ & $\mathrm{N} / \mathrm{Nm}$ \\
\hline $\begin{array}{l}\text { A } \\
\text { Hinge-Clamp }\end{array}$ & & $\begin{array}{c}0.0440 \\
(0.0440)\end{array}$ & $\begin{array}{l}2.58 \\
(2.27)\end{array}$ & $\begin{array}{l}18.40 \\
(18.51)\end{array}$ & $\begin{array}{l}-16.63 \\
(-16.63)\end{array}$ \\
\hline $\begin{array}{l}\text { B } \\
\text { Clamp-Slider/Hinge* }\end{array}$ & & $\begin{array}{c}0.0435 \\
(0.0434)\end{array}$ & $\begin{array}{c}3.33 \\
(1.68)\end{array}$ & $\begin{array}{c}9.96 \\
(11.02)\end{array}$ & $\begin{array}{c}+6.61 \\
(+6.65)\end{array}$ \\
\hline Clamp-Clamp & $\begin{array}{l}1 \\
2\end{array}$ & $\begin{array}{l}0.0419 \\
0.0433\end{array}$ & $\begin{array}{l}1.23 \\
0.94\end{array}$ & $\begin{array}{l}29.15 \\
29.69\end{array}$ & $\begin{array}{l}-4.54 \\
-4.22\end{array}$ \\
\hline Hinge-Clamp & $\begin{array}{l}1 \\
2\end{array}$ & $\begin{array}{l}0.0413 \\
0.0454\end{array}$ & $\begin{array}{l}5.11 \\
3.77\end{array}$ & $\begin{array}{l}17.03 \\
20.62\end{array}$ & $\begin{array}{l}-18.29 \\
-16.56\end{array}$ \\
\hline Clamp-Hinge & $\begin{array}{l}1 \\
2\end{array}$ & $\begin{array}{l}0.0462 \\
0.0455\end{array}$ & $\begin{array}{l}3.43 \\
1.98\end{array}$ & $\begin{array}{l}21.59 \\
26.71\end{array}$ & $\begin{array}{l}-6.57 \\
+6.39\end{array}$ \\
\hline Hinge-Hinge* & \begin{tabular}{l|l|}
1 \\
2
\end{tabular} & $\begin{array}{l}0.0410 \\
0.0443\end{array}$ & $\begin{array}{l}4.34 \\
5.50\end{array}$ & $\begin{array}{l}21.03 \\
19.67\end{array}$ & $\begin{array}{l}-9.67 \\
-8.78\end{array}$ \\
\hline Slider-Hinge* & $\begin{array}{l}1 \\
2\end{array}$ & $\begin{array}{l}0.0500 \\
0.0511\end{array}$ & $\begin{array}{l}1.60 \\
0.34\end{array}$ & $\begin{array}{l}11.63 \\
22.61\end{array}$ & $\begin{array}{l}-17.74 \\
-11.34\end{array}$ \\
\hline Clamp-Slider* & $\begin{array}{l}1 \\
2\end{array}$ & $\begin{array}{l}0.0574 \\
0.0723\end{array}$ & $\begin{array}{l}0.74 \\
0.94\end{array}$ & $\begin{array}{l}14.71 \\
13.53\end{array}$ & $\begin{array}{l}+5.93 \\
+6.09\end{array}$ \\
\hline Hinge-Slider* & $\begin{array}{l}1 \\
2\end{array}$ & $\begin{array}{l}0.0495 \\
0.0443\end{array}$ & $\begin{array}{l}8.39 \\
1.45\end{array}$ & $\begin{array}{l}21.43 \\
35.14\end{array}$ & $\begin{array}{l}-8.46 \\
-5.06\end{array}$ \\
\hline Clamp-Slider/Hinge* & $\begin{array}{l}1 \\
2\end{array}$ & $\begin{array}{l}0.0392 \\
0.0379\end{array}$ & $\begin{array}{l}1.96 \\
1.36\end{array}$ & $\begin{array}{l}12.26 \\
12.91\end{array}$ & $\begin{array}{l}+5.84 \\
+5.66\end{array}$ \\
\hline Slider/Hinge-Hinge* & $\begin{array}{l}1 \\
2\end{array}$ & $\begin{array}{l}0.0401 \\
0.0417\end{array}$ & $\begin{array}{c}28.94 \\
25.1\end{array}$ & $\begin{array}{l}10.62 \\
11.24\end{array}$ & $\begin{array}{l}-16.86 \\
-16.77\end{array}$ \\
\hline Hinge-Slider/Hinge* & $\begin{array}{l}1 \\
2\end{array}$ & $\begin{array}{l}0.0377 \\
0.0372\end{array}$ & $\begin{array}{c}8.73 \\
21.85\end{array}$ & $\begin{array}{l}13.98 \\
19.85\end{array}$ & $\begin{array}{l}-9.48 \\
-7.39\end{array}$ \\
\hline
\end{tabular}

*feasible results only for beam support conditions with finite stiffness values.

The entire assembly rests on a rotary stage, that can be used to set the horizontal flexion/extension angle $\gamma$. A separate frame serves as the mount for the prototype's back interfaces and the torso reference shell.

Prototype A consists of three major parts. The first part of the flexure containing the back interface, is simply bolted to the frame. It is connected to the second part of the flexure via a flange, which is bolted and reinforced with instant adhesive. At its other end, the second part of the flexure is mounted via a shaft to a ball bearing which is contained in the the arm interface. This hinged interface in turn is bolted to the arm shells via two slotted holes.

Prototype B, shown in Figure 2 features a more complex design. A shuttle which incorporates a sliding block, bolts for connecting springs and counterweights, as well as a shaft supported by two ball bearings constitutes the back interface of this design. The sliding block runs in a linear track, which is fixed to a long aluminum profile that is attached to the frame. To set the bi-directional stiffness of the translational degree of freedom two tension springs are used. The main spring covers most of the range of motion and is fixed between the lower end of the aluminum profile and the shuttle. The secondary spring is suspended from the top of the profile and connected to the shuttle via a string which disengages the spring when not in use. In addition to the springs, another string connects the shuttle to a counterweight, which is suspended from a pulley at the top of the aluminum profile. It is used to adjust the effective weight of the shuttle assembly to the value set in the simulation. The first part of the flexure is fixed to the shaft of the shuttle and connects to the second part of the flexure in the same way as prototype A. That second part containing the arm interface is then directly fixed to the arm shells.

The entire setup is controlled and the sensor readings obtained via Simulink Real-Time. To this end a PC with a National Instruments PCI-6221 multifunction I/O card and a National Instruments BNC-2110 adapter are used. During experiments the arm is raised from the lowest position $\theta=0$ to the raised position $\theta=\pi$ and back to the lowest position using a constant speed. The signals were acquired at a rate of $100 \mathrm{~Hz}$ and the force signal was filtered using a moving average filter with a window size of 20 samples. Each configuration was measured with and without the flexure. The two measurements were then subtracted from each other to isolate the mechanical behavior of the flexure mechanism. Multiplying the force data with the partial diameter of the timing pulley at the arm yields the shoulder moment. In the absence of an absolute zero reference for the force signal, the moment offset has been adjusted such that the zero crossing for the unstressed state at which the mechanism was assembled matches the simulation.

\section{RESUlts}

A total number of 1616 optimizations were conducted, of which 204 resulted in feasible mechanism designs. These were found for 18 of the 25 investigated support conditions. All support conditions yielded feasible results for finite joint stiffnesses. However, only three achieved a balancing behavior below the threshold of NMEA $\leq 0.05$ when considering no stiffness at the released joints.

We categorized each of the feasible mechanisms by the amount of displacement displayed at each joint. Accordingly, the label Clamp, Slider, Hinge or Slider/Hinge was assigned to each interface. Thereby we found 10 feasible categories of mechanisms that correspond to the 16 possible kinematic combinations shown in Table I. The other 6 kinematic combinations were avoided in optimization by assigning high stiffnesses to some of the released joints. Consequently, these joints would display no significant motion, making them behave as if rigid or absent. Out of each feasible category two designs were chosen as representative examples, and dimensioned for an amplitude of $10 \mathrm{Nm}$. Animations of these two examples, labeled 1 and 2, for each category can be seen in Figure 3. The categories are named by first stating the condition at the arm interface and then, separated by a dash, at the back interface. The three kinematic categories that work without considering finite stiffnesses are the first three shown in Figure 3. They feature either two clamped interfaces, or one clamped and one hinged interface. The chosen examples for these three categories all feature approximately zero stiffness in the joints. All other categories required finite joint stiffnesses in order to work. Some rely solely on the interfaces for their function, using the flexure as a structural element. An example for this is the Slider/Hinge-Hinge category in which both designs resemble the inverse slider-crank mechanism described by Kim et al. [36].

The Hinge-Clamp and the Clamp-Slider/Hinge categories were chosen for further investigation. A was obtained by reoptimizing the Hinge-Clamp category with no hinge stiffness. 

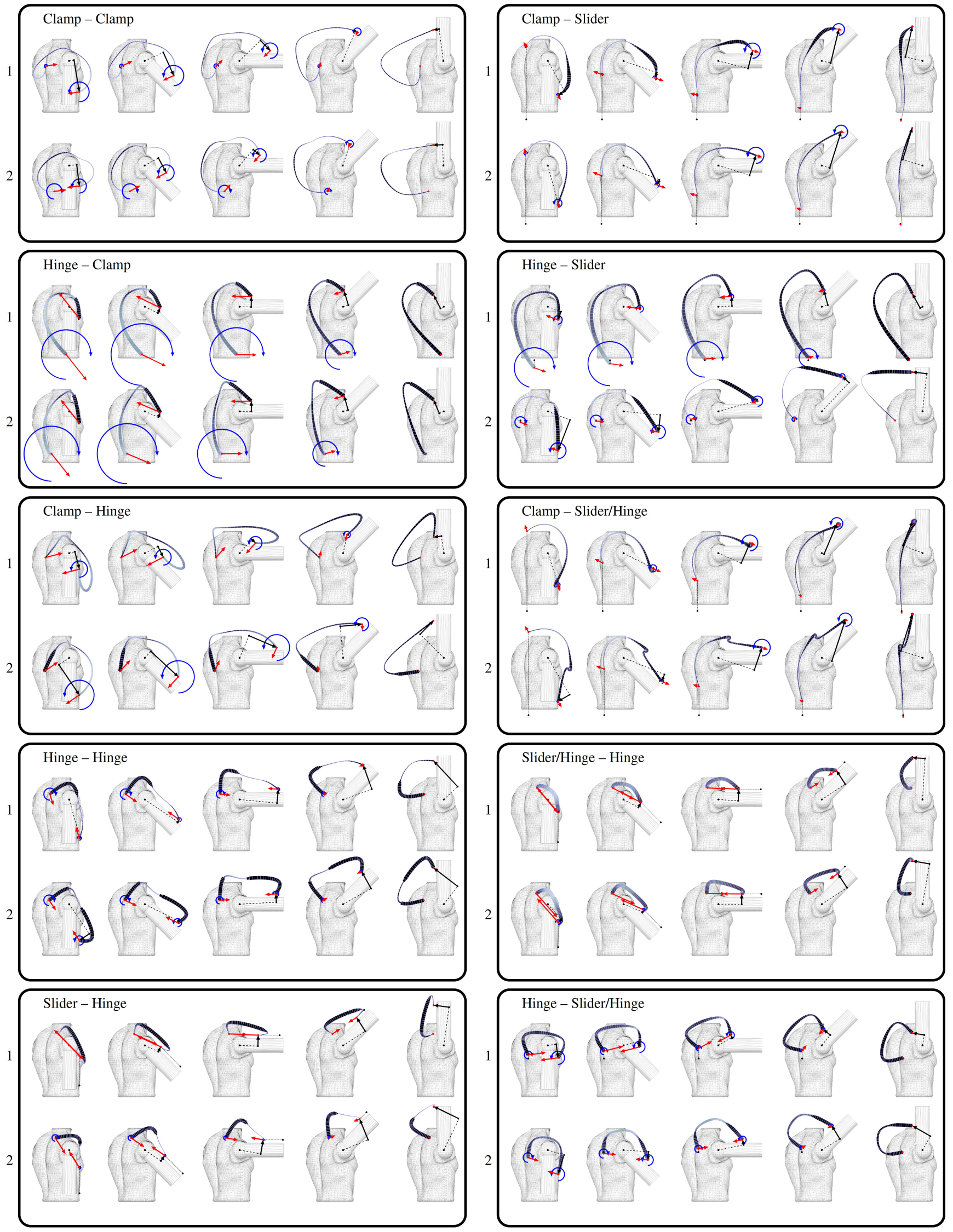

Fig. 3. Animations of the example mechanisms arranged by kinematic category. Red arrows are proportional to reaction forces $(1 \mathrm{~mm} / \mathrm{N})$, the blue arrow's radii to reaction moments $(33 \mathrm{~mm} / \mathrm{Nm})$. Black arrows depict the lever arm of the arm interface force with respect to the shoulder joint, marked by a dot. A perpendicular dashed line connects the lever arm arrow and shoulder joint. Dotted lines show slider displacements from the initial positions marked by dots. 
a)

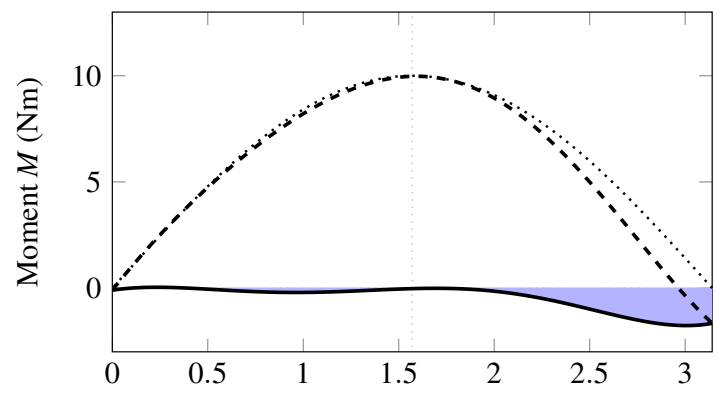

b)

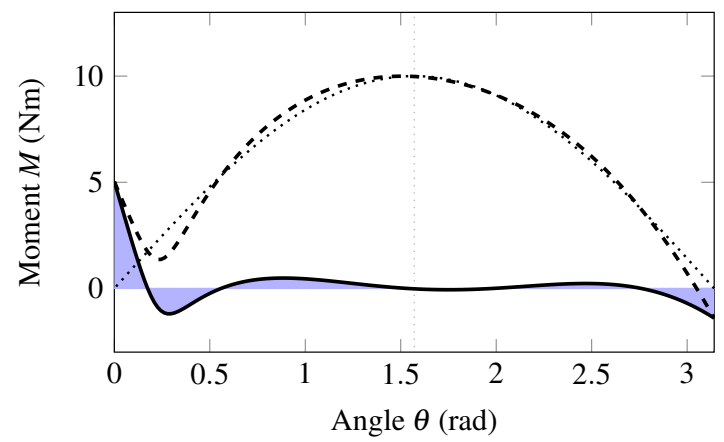

Fig. 4. Optimized balancing behavior of a) $\mathbf{A}$ and b) $\mathbf{B}$. The dashed line depicts the computed moment generated by the mechanism. The dotted line shows the ideal characteristic, i.e. the inverse of the load moment. The solid line shows the balanced behavior of the mechanism, when adding the load moment to the mechanism's moment. This diagram shows good balancing behavior for both prototypes throughout the range of motion. A shows progressively imbalanced behavior towards the raised position, while B shows a sudden deviation at smaller angles.

For $\mathbf{B}$ the stiffness of the back hinge was set to zero, while the back slider was assigned a constant stiffness of $100 \mathrm{~N} / \mathrm{m}$, when re-optimizing the Clamp-Slider/Hinge category. The prototypes for $\mathbf{A}$ and $\mathbf{B}$ were accidentally dimensioned only on the basis of the stresses at the last load step. Consequently, they surpassed the stress limit by $1.9 \mathrm{MPa}$ and $10.8 \mathrm{MPa}$, respectively, when considering the entire range of motion. However, this did not lead to failure or any other observed negative consequences. To provide an accurate comparison with the other examples, corrected values for beam dimensions observing the stress limit are given in brackets.

A comparison between the computed performance metrics of the prototypes and the two examples of each kinematic category is given in Table II. Since the mechanisms in this study are all designed for a moment amplitude of $10 \mathrm{Nm}$, the relative shear force represents $10 \%$ of the absolute maximum shear force. Graphs showing the optimized angle-dependent balancing behavior for $\mathbf{A}$ and $\mathbf{B}$ are shown in Figure 4 . Corresponding graphs for all other examples are provided in supplementary file 1 . The construction parameters for the flexures employed in $\mathbf{A}$ and $\mathbf{B}$ are given in Table III in the appendix. Corresponding tables for all other examples along with information about the joint stiffnesses and ranges of motion are provided in supplementary file 2. Movies showing the full animations of all simulated mechanisms shown in juxtaposition to the balancing graphs are provided in supplementary file 3 . Table IV shows the energy distribution between the flexure and the individual joints at $\theta=0$. a)

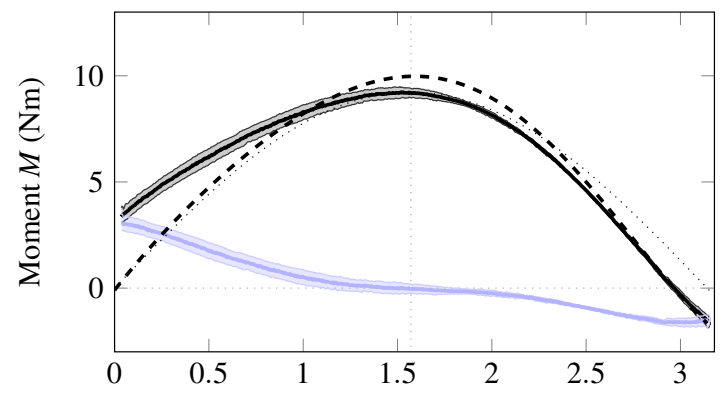

b)

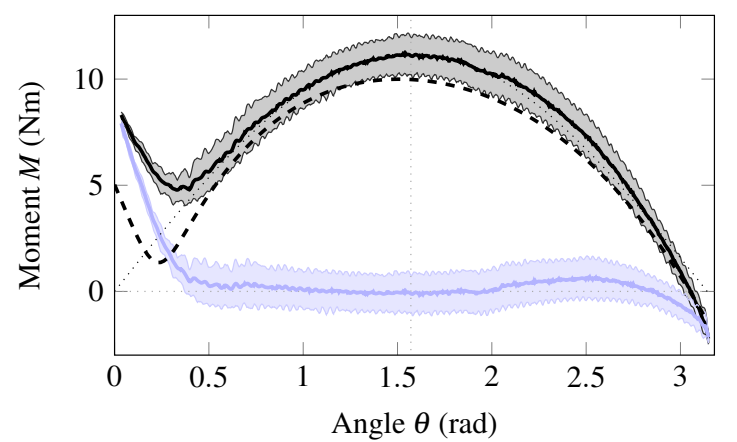

Fig. 5. Experimental results for a) prototype $\mathbf{A}$ and b) prototype $\mathbf{B}$. The thin black solid line depicts the measured moment exerted by the mechanism. The thick black solid line depicts the mean and the shaded area in between indicates the hysteresis. A sine curve matching the maximum amplitude of the measured mean is depicted by the black thin dotted line. The balanced moment characteristic, i.e. the difference between the sine curve and measured data, is analogously depicted in light blue. The corresponding simulation result is shown by the dashed line. In comparison to the computed results, prototype A shows additional progressively imbalanced behavior towards the smaller angles. For prototype B the sudden deviation from balanced behavior at smaller angles occurs earlier. Furthermore, both results show a mismatch in amplitude of $-0.78 \mathrm{Nm}$ and $+1.2 \mathrm{Nm}$ for $\mathbf{A}$ and $\mathbf{B}$, respectively. Prototype $\mathbf{B}$ exhibits significantly more hysteresis than prototype $\mathbf{A}$.

The results of measurements performed at $\frac{\pi}{60} \mathrm{rad} / \mathrm{sec}$ are shown in Figure 5. The animations of $\mathbf{A}$ and $\mathbf{B}$ in juxtaposition to the photographs of the experiment are shown in Figure 6. Corresponding, movies showing the full animation and photo time lapse, alongside the respective graphs are provided in the supplementary files 4 and 5. Movies showing the experiment of $\mathbf{A}$ and $\mathbf{B}$ from several different angles are provided in the supplementary files 6 and 7.

\section{Discussion}

A surprisingly large variety of different mechanisms that fulfill both the requirements regarding gravity balancing and collision avoidance was found. These can be categorized by their apparent kinematic behavior as shown in Figure 3, but also by they way they achieve gravity balancing and the degree to which they utilize the flexure.

When considering the force and moment at the arm interface as can be seen in Figure 3, there are three ways of achieving gravity balancing. Mechanisms that feature no significant moment at the arm can only utilize the interface force for balancing. At the raised position they start with a small force, but large lever arm. As the arm moves down the force increases while the lever arm decreases, creating the sineshaped moment at the shoulder joint. At the lowest position the force points directly at the shoulder joint to achieve a 

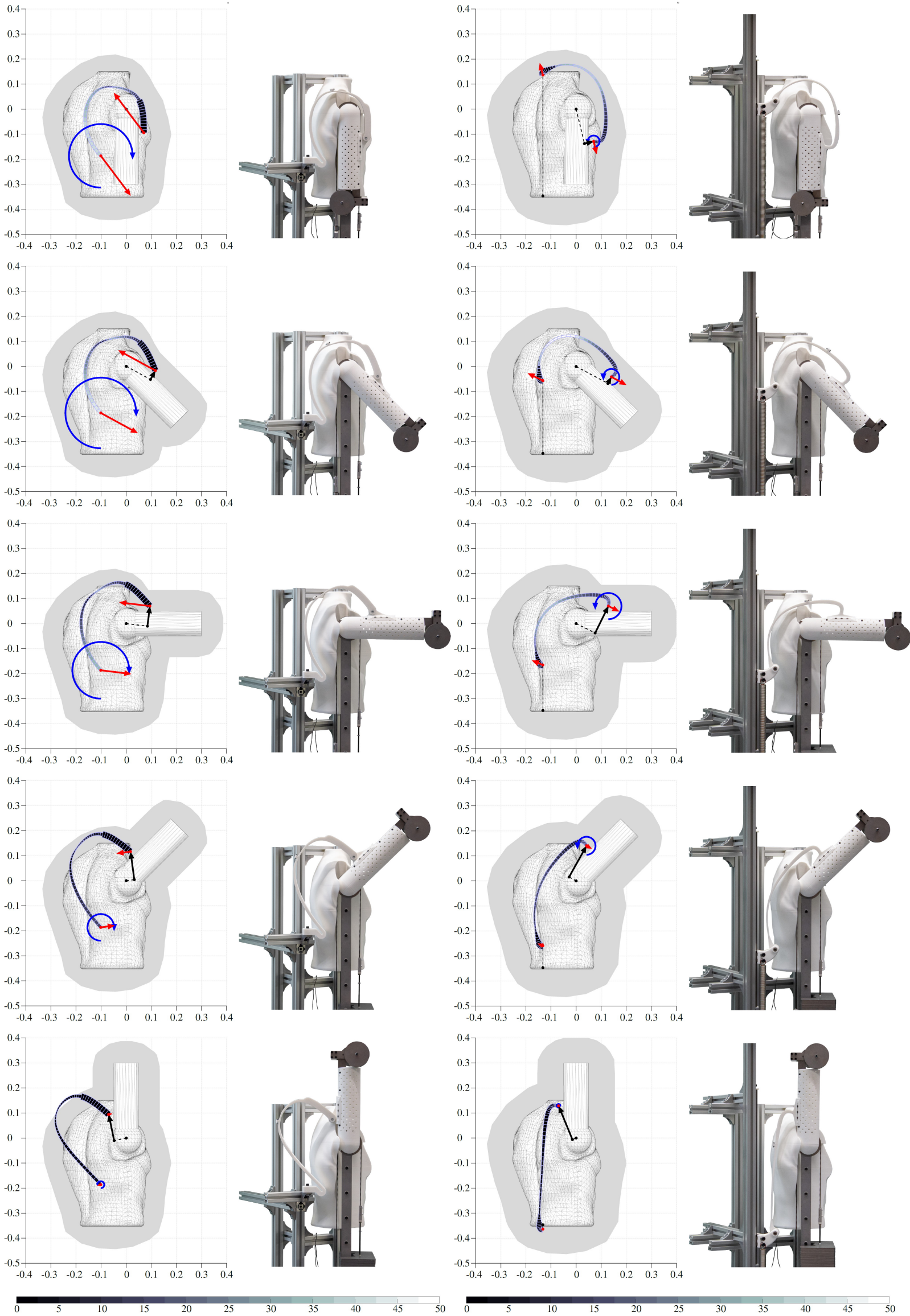

Fig. 6. Juxtaposition of animations and photographs from the experiment for a) $\mathbf{A}$ and b) $\mathbf{B}$. Axes are in $\mathrm{m}$, color scale in MPa. The remaining description of the animations is equal to Figure 3. The shuttle counterweight and secondary spring were removed from b), as not to obstruct the view on the flexure. 
zero moment. Examples for this behavior are the categories Hinge-Clamp, Slider-Hinge and Slider/Hinge-Hinge. As for their functioning the force vector has to stay mostly parallel to the arm, these three categories feature larger relative shear forces than all other categories. Mechanisms that feature a progressively increasing moment towards lower angles, have to use the interface force to counteract it. As the arm moves from the raised position to the lowered position the moment arm is increasing in opposite direction, often flipping its orientation relative to the shoulder. At lower angles the force vector points mostly perpendicular to the arm. Examples for this are the categories Clamp-Clamp, Clamp-Hinge, Hinge-Slider and Hinge-Slider/Hinge. Lastly, mechanisms that can modulate the moment at the arm interface, can achieve balancing irrespective of the force. For this type of behavior the location of the back interface has to change significantly. Consequently, a slider with large stroke at the back is required. Examples for this are the Clamp-Slider and Clamp-Slider/Hinge categories. These type of mechanisms have the lowest interface forces.

When considering the energy distribution shown in Table IV we can, again, divide the mechanisms into three different types. The first type uses only the flexure for energy storage. It corresponds to the three categories Clamp-Clamp, Hinge-Clamp and Clamp-Hinge for which results were found, when no joint stiffness was considered. The second type is a hybrid which uses both the flexure and interfaces for storing energy. Mechanisms of this type feature up to two joints, of which one is used for energy storage. They belong to the categories Clamp-Slider, Hinge-Slider and ClampSlider/Hinge. The third type nearly exclusively uses the joints for energy storage. In these cases the flexure is reduced to a means of transmission. For that reason this type was not considered for further investigation in this study. This pertains to the categories Hinge-Hinge, Slider-Hinge, Slider/HingeHinge and Hinge-Slider/Hinge.

For A the Hinge-Clamp category was chosen, as one of the designs that utilizes only the flexure for energy storage. It, therefore, offered low complexity and a small number of parts. In comparison to the Clamp-Clamp and Clamp-Hinge categories it showed the best potential for close-to-body alignment and relative strength. Though the maximum protrusion is relatively high, it drops steadily from $18 \mathrm{~cm}$ at $\theta=\pi$, below $10 \mathrm{~cm}$ at $\theta=\frac{\pi}{2}$ and down to $6 \mathrm{~cm}$ at $\theta=0$. However, the large shear force at the arm and very large moment at the back interface are the two main disadvantages of this design. In addition, the experiments indicate that purely force based gravity balancing may be more sensitive to disturbances. Due to the small lever arm the orientation of the force vector with respect to the shoulder has to be very precise in order to function properly. Our results indicate that with increasing stress and deformation in the system, this may be hard to achieve causing the progressive deviation between simulation and experiments towards smaller angles, as shown in Figure 5. Therefore, in retrospect, the Clamp-Hinge category may be the better choice out of the flexure-only designs.

For $\mathbf{B}$ we chose the Clamp-Slider/Hinge category. As one of the hybrid types it is more complex, but offered the best theoretical performance out of the flexure-based designs. In addition, the way the mechanism aligns with the body and the low reaction forces and moments seemed advantageous. In comparison to $\mathbf{A}$ the protrusion remains more steady, dropping from $10 \mathrm{~cm}$ at $\theta=\pi$ down to $7 \mathrm{~cm}$ for the range between $\theta=2$ to $\theta=0.5$ and going back up to $10 \mathrm{~cm}$ at $\theta=0$. In comparison to the very similar Clamp-Slider category the additional hinge at the back yields performance gains throughout all metrics. By observation of the energy distribution and stiffnesses, we concluded that for this prototype design we could assign predefined stiffness values to the joints. Thereby, we reduced the search space in the simulation and simplified the construction. Considering the measurement data shown in Figure 5 the balancing behavior seems more robust than that of prototype $\mathbf{A}$, showing larger qualitative divergence only for angles below $0.5 \mathrm{rad}$. At these smaller angles also a positional deviation of the back interface between simulation and experiments occurs, as can be seen in Figure 6. However, this is in part caused by the missing counterweight.

All mechanisms found in this paper exhibit a maximum protrusions of at least $10 \mathrm{~cm}$. Hence, modifications to the synthesis method are likely required to further improve the alignment with the body and reduce protrusion. The jointcentric designs may be improved by replacing the flexure element by a structure or mechanism which aligns laterally to the body, as is done in the ShoulderX [22] which shares similarities with the Slider/Hinge-Slider designs. For the flexure-based designs the Clamp-Slider/Hinge category seems to offer the best prospects. Potential ways of improving the alignment to the body may include investigation of nonlinear tracks for the slider that conform better to the body, actively guiding the flexure, e.g. with rollers, allowing contact with the body and the investigation of spatial flexure designs that connect laterally to the arm. In addition, the balancing error at lower angles needs to be addressed, and though out-of-plane behavior was not explicitly investigated, a vertical pivot to facilitate horizontal flexion/extension will likely be required. In preparation to this study contact with the body and spatial flexure designs have been investigated. However, the high computational cost for the contact analysis and the largely increased search space when considering additional bending and torsion deformation angles of the flexure were reasons to exclude these aspects from this study.

\section{CONCLUSION}

With this paper we set out to find a close-to-the-body gravity balancing flexure-based mechanism for shoulder support that may be used without any additional rigid linkages between the torso and the arm. A broad optimization study into different kinematic support conditions revealed a multitude of possible designs - many of which we left for further investigation.

Of the two designs that we developed into prototypes and evaluated in experiment both show good gravity balancing behavior. Prototype $\mathbf{A}$ reduces the required maximum moment to lift the arm by more than $80 \%$ over the entire simulated and $85 \%$ of the measured range of motion. Prototype B shows even better balancing qualities, offering the same more-than- $80 \%$ reduction over $97 \%$ of the simulated and 
$92 \%$ of the measured range of motion. In addition, design $\mathbf{B}$, having the lowest protrusion out of all flexure-based designs, features a maximum protrusion that is $46 \%$ less than that of design A. Furthermore, the low interface forces and moments of design $\mathbf{B}$ which are over $60 \%$ lower compared to design A are potential key enablers for a compliant exoskeleton structure. Therefore, a design with a spring loaded slider and a hinge at the back of the wearer and a tapered flexure connecting it to the upper arm appears to be the most promising solution for the proposed exoskeleton architecture.

Though we believe that in terms of size and protrusion the current design $\mathbf{B}$ could already compete with the mechanisms employed in commercially available shoulder supports, our device is still far from the aspired $3 \mathrm{~cm}$ distance to the body. We provided possible leads to mitigate this shortcoming in future work. Aside from this, additional work will be necessary to translate the test bench prototype mechanism into a wearable assistive device. To this end, better integration, simplification and customization of all components will be the main challenges.

\section{APPENDIX A}

\section{SUPPLEMENTARY FILES}

\section{Supplementary file 1 - Additional_Graphs.pdf}

Graphs showing the angle-dependent balancing behavior for all example mechanisms, arranged by their kinematic categories.

\section{Supplementary file 2 - Additional_Tables.pdf}

A table stating the flexure parameters and a table stating the joint stiffnesses and joint ranges of motion for all example mechanisms.

Supplementary file 3 - Simulations.mp4

Movie showing the full animations of all simulated mechanisms shown in Figure 3, in juxtaposition to the graphs from supplementary file 1.

\section{Supplementary file 4 - Prototype_A.mp4}

Movie showing the full animation and photo time lapse corresponding to Figure 6, in juxtaposition to the graphs in Figure 4 and 5 for design A.

\section{Supplementary file 5 - Prototype B.mp4}

Movie showing the full animation and photo time lapse corresponding to Figure 6, in juxtaposition to the graphs in Figure 4 and 5 for design B.

Supplementary file 6 - Prototype_A_Experiment.mp4

Movie showing the experiment of $\mathbf{A}$ from several different angles.

Supplementary file 7 - Prototype_B_Experiment.mp4

Movie showing the experiment of $\mathbf{B}$ from several different angles.

\section{APPENDIX B}

TABLES

TABLE III

FLEXURE PARAMETERS

\begin{tabular}{|c|c|c|c|c|c|}
\hline Parameter & Unit & \multicolumn{2}{|c|}{$\mathbf{A}$} & \multicolumn{2}{|c|}{ B } \\
\hline$s_{x}$ & $\mathrm{~mm}$ & \multicolumn{2}{|c|}{-101.4} & \multicolumn{2}{|c|}{-132.6} \\
\hline$s_{z}$ & $\mathrm{~mm}$ & \multicolumn{2}{|c|}{-186.4} & \multicolumn{2}{|c|}{-346.6} \\
\hline$l_{1}$ & $\mathrm{~mm}$ & \multicolumn{2}{|c|}{309.6} & \multicolumn{2}{|c|}{13.5} \\
\hline$l_{2}$ & $\mathrm{~mm}$ & \multicolumn{2}{|c|}{127.8} & \multicolumn{2}{|c|}{468} \\
\hline$l_{3}$ & $\mathrm{~mm}$ & \multicolumn{2}{|c|}{155.2} & \multicolumn{2}{|c|}{42.4} \\
\hline$\varphi_{1}$ & $\mathrm{rad}$ & \multicolumn{2}{|c|}{2.145} & \multicolumn{2}{|c|}{2.666} \\
\hline$\varphi_{2}$ & $\mathrm{rad}$ & \multicolumn{2}{|c|}{0.9105} & \multicolumn{2}{|c|}{1.4635} \\
\hline$\varphi_{3}$ & $\mathrm{rad}$ & \multicolumn{2}{|c|}{-0.4639} & \multicolumn{2}{|c|}{0.2735} \\
\hline$t_{1}$ & $\mathrm{~mm}$ & 12.5 & (12) & 47.9 & (38.1) \\
\hline$t_{2}$ & $\mathrm{~mm}$ & 11 & (10.6) & 5.2 & $(4.2)$ \\
\hline$t_{3}$ & $\mathrm{~mm}$ & 4.4 & $(4.2)$ & 8 & (6.3) \\
\hline$t_{4}$ & $\mathrm{~mm}$ & 12.9 & (12.4) & 16.1 & (12.8) \\
\hline$t_{5}$ & $\mathrm{~mm}$ & 197.6 & (189.4) & 12.2 & $(9.7)$ \\
\hline$w$ & $\mathrm{~mm}$ & 38.7 & $(44.1)$ & 30 & (59.5) \\
\hline
\end{tabular}

TABLE IV

ENERGY DISTRIBUTION AT $\theta=0$ ON A PERCENTAGE BASIS

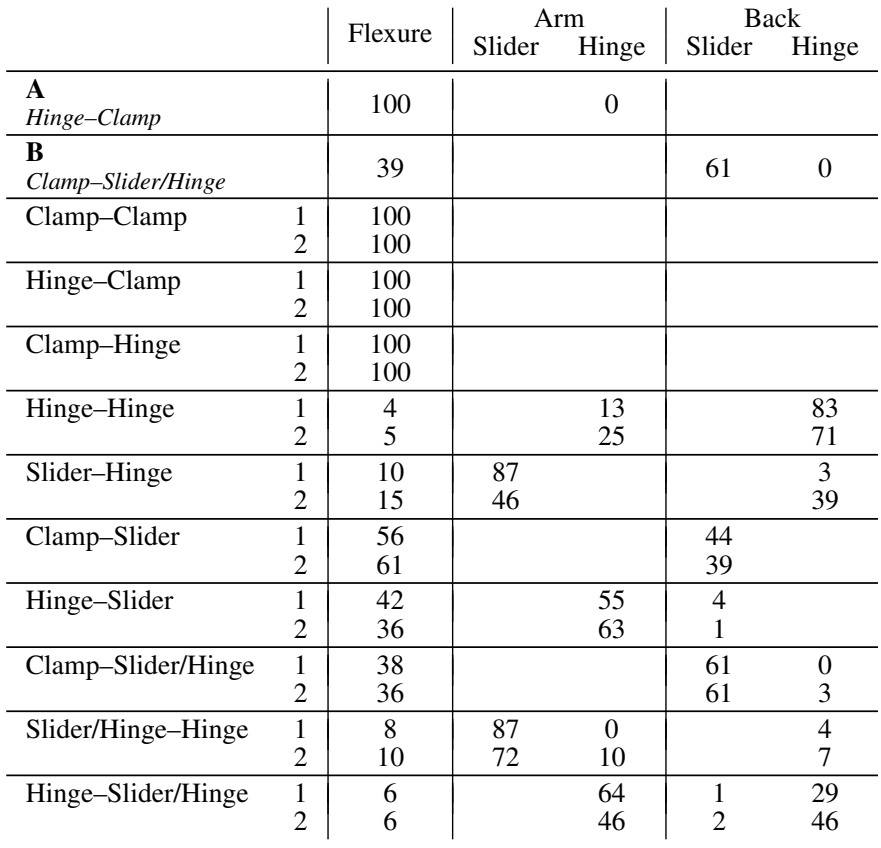

\section{REFERENCES}

[1] L. A. van der Heide, B. van Ninhuijs, A. Bergsma, G. J. Gelderblom, D. J. van der Pijl, and L. P. de Witte, "An overview and categorization of dynamic arm supports for people with decreased arm function," Prosthetics and Orthotics International, vol. 38, no. 4, pp. 287-302, 2014, PMID: 23950551.

[2] A. G. Dunning and J. L. Herder, "A review of assistive devices for arm balancing," in 2013 IEEE 13th International Conference on Rehabilitation Robotics (ICORR), Jun. 2013, pp. 1-6.

[3] T. McFarland and S. Fischer, "Considerations for industrial use: A systematic review of the impact of active and passive upper limb exoskeletons on physical exposures," IISE Transactions on Occupational Ergonomics and Human Factors, vol. 7, no. 3-4, pp. 322-347, 2019.

[4] P. Maciejasz, J. Eschweiler, K. Gerlach-Hahn, A. Jansen-Troy, and S. Leonhardt, "A survey on robotic devices for upper limb rehabilitation," Journal of NeuroEngineering and Rehabilitation, vol. 11, no. 1, Jan. 2014, Art. no. 3.

[5] S. K. Manna and V. N. Dubey, "Comparative study of actuation systems for portable upper limb exoskeletons," Medical Engineering \& Physics, vol. 60 , pp. $1-13,2018$.

[6] R. J. Varghese, D. Freer, F. Deligianni, J. Liu, and G.-Z. Yang, "Chapter 3 - Wearable robotics for upper-limb rehabilitation and assistance: A review of the state-of-the-art, challenges, and future research," in Wearable Technology in Medicine and Health Care, R. K.-Y. Tong, Ed. Academic Press, 2018, pp. 23 - 69.

[7] A. J. Veale and S. Q. Xie, "Towards compliant and wearable robotic orthoses: A review of current and emerging actuator technologies," Medical Engineering \& Physics, vol. 38, no. 4, pp. 317 - 325, 2016.

[8] P. N. Kooren, A. G. Dunning, M. M. H. P. Janssen, J. Lobo-Prat, B. F. J. M. Koopman, M. I. Paalman, I. J. M. de Groot, and J. L. Herder, "Design and pilot validation of a-gear: a novel wearable dynamic arm support," Journal of NeuroEngineering and Rehabilitation, vol. 12, no. 1, p. 83, Sep 2015.

[9] J. Hull, R. Turner, A. A. Simon, and A. T. Asbeck, "A novel method and exoskeletons for whole-arm gravity compensation," IEEE Access, vol. 8, pp. $143144-143159,2020$.

[10] J. Chen and P. S. Lum, "Pilot testing of the spring operated wearable enhancer for arm rehabilitation (springwear)," Journal of NeuroEngineering and Rehabilitation, vol. 15, no. 1, p. 13, Mar 2018.

[11] EksoVest ${ }^{\mathrm{TM}}, E V O$ \& EksoUE, Ekso Bionics ${ }^{\circledR}$, Richmond, CA, USA, https://eksobionics.com/.

[12] Wilmington Robotic Exoskeleton (WREX), JAECO Orthopedic, Hot Springs, AR, USA, https://jaecoorthopedic.com/.

[13] T. Haumont, T. Rahman, W. Sample, M. Marnie King, C. Church, J. Henley, and S. Jayakumar, "Wilmington robotic exoskeleton: A novel device to maintain arm improvement in muscular disease," Journal of Pediatric Orthopaedics, vol. 31, 2011. 
[14] Airframe ${ }^{\mathrm{TM}}$, Levitate Technologies, San Diego, CA, USA, https://www.levitatetech.com/.

[15] S. Iranzo, A. Piedrabuena, D. Iordanov, U. Martinez-Iranzo, and J.-M. Belda-Lois, "Ergonomics assessment of passive upper-limb exoskeletons in an automotive assembly plant," Applied Ergonomics, vol. 87, p. 103120, 2020.

[16] S. Kim, M. A. Nussbaum, M. I. Mokhlespour Esfahani, M. M. Alemi, S. Alabdulkarim, and E. Rashedi, "Assessing the influence of a passive, upper extremity exoskeletal vest for tasks requiring arm elevation: Part I - "expected" effects on discomfort, shoulder muscle activity, and work task performance," Applied Ergonomics, vol. 70, pp. 315 - 322, 2018.

[17] MATE, Comau, Turin, Italy, https://www.comau.com/.

[18] I. Pacifico, A. Scano, E. Guanziroli, M. Moise, L. Morelli, A. Chiavenna, D. Romo, S. Spada, G. Colombina, F. Molteni, F. Giovacchini, N. Vitiello, and S. Crea, "An experimental evaluation of the proto-MATE: A novel ergonomic upper-limb exoskeleton to reduce workers' physical strain," IEEE Robotics Automation Magazine, vol. 27, no. 1, pp. 54-65, 2020.

[19] PAEXO, Ottobock, Duderstadt, Germany, https://paexo.com/.

[20] P. Maurice, J. Čamernik, D. Gorjan, B. Schirrmeister, J. Bornmann, L. Tagliapietra, C. Latella, D. Pucci, L. Fritzsche, S. Ivaldi, and J. Babič, "Objective and subjective effects of a passive exoskeleton on overhead work," IEEE Transactions on Neural Systems and Rehabilitation Engineering, vol. 28, no. 1, pp. 152-164, 2020.

[21] ShoulderX, SuitX, Emeryville, CA, USA, https://www.suitx.com/.

[22] L. Van Engelhoven and H. Kazerooni, "Design and intended use of a passive actuation strategy for a shoulder supporting exoskeleton," in 2019 Wearable Robotics Association Conference (WearRAcon), 2019, pp. 7-12.

[23] SkelEx, Skel-Ex, Rotterdam, The Netherlands, https://www.skelex.com/.

[24] A. de Vries, M. Murphy, R. Könemann, I. Kingma, and M. de Looze, "The amount of support provided by a passive arm support exoskeleton in a range of elevated arm postures," IISE Transactions on Occupational Ergonomics and Human Factors, vol. 7, no. 3-4, pp. 311-321, 2019.

[25] M. Tschiersky, E. E. G. Hekman, D. M. Brouwer, and J. L. Herder, "Gravity balancing flexure springs for an assistive elbow orthosis," IEEE Transactions on Medical Robotics and Bionics, vol. 1, no. 3, pp. 177188, Aug 2019.

[26] Z. Cheng, S. Foong, D. Sun, and U. X. Tan, "Algorithm for design of compliant mechanisms for torsional applications," in 2014 IEEE/ASME International Conference on Advanced Intelligent Mechatronics, July 2014, pp. 628-633.

[27] — " "Towards a multi-DOF passive balancing mechanism for upper limbs," in 2015 IEEE International Conference on Rehabilitation Robotics (ICORR), Aug. 2015, pp. 508-513.

[28] G. Radaelli and J. L. Herder, "Isogeometric shape optimization for compliant mechanisms with prescribed load paths," in ASME 2014 International Design Engineering Technical Conferences and Computers and Information in Engineering Conference (DETC2014), vol. 5A: 38th Mechanisms and Robotics Conference, 2014.

[29] _ "A monolithic compliant large-range gravity balancer," Mechanism and Machine Theory, vol. 102, no. Supplement C, pp. 55 - 67, 2016

[30] — "Shape optimization and sensitivity of compliant beams for prescribed load-displacement response," Mechanical Sciences, vol. 7, no. 2, pp. 219-232, 2016

[31] P. de Leva, "Adjustments to Zatsiorsky-Seluyanov's segment inertia parameters," Journal of Biomechanics, vol. 29, no. 9, pp. 1223-1230, 1996.

[32] E. Rocon, A. F. Ruiz, R. Raya, A. Schiele, J. L. Pons, J. M. BeldaLois, R. Poveda, M. J. Vivas, and J. C. Moreno, Human-Robot Physical Interaction. John Wiley \& Sons, Ltd, 2008, ch. 5, pp. 127-163.

[33] A. Oosterwijk, M. Nieuwenhuis, C. van der Schans, and L. Mouton, "Shoulder and elbow range of motion for the performance of activities of daily living: A systematic review," Physiotherapy Theory and Practice, vol. 34, no. 7, pp. 505-528, 2018, PMID: 29377745.

[34] J. B. Jonker and J. P. Meijaard, SPACAR - Computer Program for Dynamic Analysis of Flexible Spatial Mechanisms and Manipulators. Berlin, Heidelberg: Springer Berlin Heidelberg, 1990, pp. 123-143.

[35] J. P. Meijaard, "Fluid-conveying flexible pipes modeled by largedeflection finite elements in multibody systems," Journal of Computational and Nonlinear Dynamics, vol. 9, no. 1555-1415, 2014.

[36] H. Kim, J. Min, and J. Song, "Multiple-degree-of-freedom counterbalance robot arm based on slider-crank mechanism and bevel gear units," IEEE Transactions on Robotics, vol. 32, no. 1, pp. 230-235, 2016.

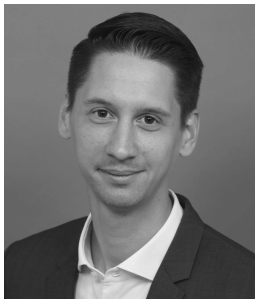

Martin Tschiersky received his B.Sc. and M.Sc. degrees in mechanical engineering from the University of Duisburg-Essen, Duisburg, Germany, in 2012 and 2014, respectively.

$\mathrm{He}$ is currently pursuing the Ph.D. degree in mechanical engineering at the University of Twente, Enschede, The Netherlands. His research interests include the development of assistive and prosthetic devices, passive gravity balancing by means of elastic energy storage and the design of flexure-based compliant mechanisms and actuators.

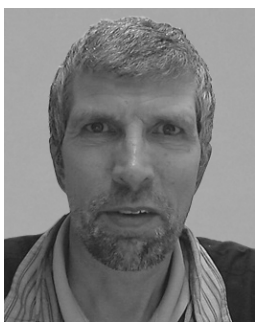

Edsko E. G. Hekman (M'18) received his B.Sc. degree in science and engineering from Calvin College, Grand Rapids, MI, USA, in 1981 and his M.Sc. degree in mechanical engineering from Delft University of Technology, Delft, The Netherlands, in 1986.

After 6 years of developing equipment for medical research at the Radboud University, Nijmegen, he joined the Research and Teaching Staff of the University of Twente, Enschede, The Netherlands. His main research interests are the development of prosthetic, orthotic and robotic devices for restoration of gait and mobility, and in general devices in the technical biomedical field.

Mr. Hekman is member of the International Society of Biomechanics.

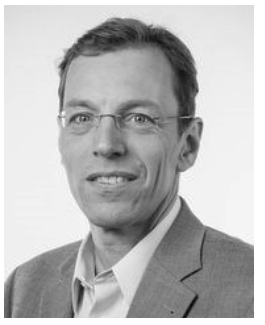

Just L. Herder (M'06) received his M.Sc. and his $\mathrm{Ph} . \mathrm{D}$. degrees in mechanical engineering from Delft University of Technology, Delft, The Netherlands, in 1992 and 2001, respectively.

$\mathrm{He}$ held visiting positions at Laval University, Canada, and at MIT, USA, as a Fulbright Visiting Scholar. Currently, he is a Full Professor and Head of the Precision and Microsystems Department at Delft University of Technology, Delft, The Netherlands. He has widely published in international peerreviewed journals and conferences and gave over a dozen keynote talks at international conferences. He holds 25 patents in different areas of mechanism design, based on which seven start-up companies have emerged.

Prof. Herder has received several international awards, including six best paper awards. He is an ASME fellow and Founding Editor-in-Chief of Mechanical Sciences.

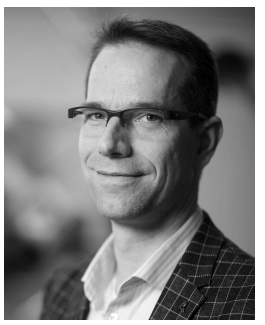

Dannis M. Brouwer received a M.Sc. degree in mechanical engineering, in 1998, and a PDEng degree in mechatronic design in 2001 from Eindhoven University of Technology, Eindhoven, The Netherlands. In 2007 he received his Ph.D. degree from the University of Twente, Enschede, The Netherlands.

$\mathrm{He}$ has 8 years of industrial experience with the Philips Centre for Industrial Technology (CFT), Eindhoven, and Demcon Advanced Mechatronics, Enschede. He is currently a Full Professor at the University of Twente, where he acts as Chair of the Precision Engineering group and as Vice-Head of the department of Mechanics of Solids Surfaces and Systems. He has (co-)authored over 40 refereed journal articles, 90 conference contributions and 8 patents on a variety of subjects including flexure mechanisms, macro and micro manipulators, variable impedance robotics and precision engineering.

Prof. Brouwer received the prestigious personal VIDI grant for his large range of motion flexure research in 2015, where he was ranked first out of 48 competitors. He has served as a Director-at-Large for the American Society for Precision Engineering and Associate Editor for Precision Engineering. 